\title{
COVID-19 and Cardiac Arrhythmias
}

\author{
Martin Duckheim ${ }^{1}$ Jürgen Schreieck ${ }^{1}$ \\ ${ }^{1}$ Innere Medizin III, Department of Cardiology and Angiology, \\ Eberhard Karls Universität Tübingen, Tübingen, Germany \\ Hamostaseologie 2021;41:372-378.
}

\begin{abstract}
Address for correspondence Martin Duckheim, MD, Innere Medizin III, Klinik für Kardiologie und Angiologie, Otfried-Müller-Str. 10, 72076 Tübingen, Germany

(e-mail: martin.duckheim@med.uni-tuebingen.de).
\end{abstract}

Abstract
Keywords
- COVID-19
- cardiac arrhythmia
- sudden cardiac death
- atrial fibrillation
- ventricular
tachycardia
- SARS-CoV-2

Zusammenfassung

Schlüsselwörter

- COVID-19

- Arrhythmien

- plötzlicher Herztod

- Vorhofflimmern

- ventrikuläre Tachykardie

- SARS-CoV-2
Since the coronavirus disease (COVID-19) pandemic spread unrelentingly all over the world, millions of cases have been reported. Despite a high number of asymptomatic cases, the course of the disease can be serious or even fatal. The affection of the myocardium, called myocardial injury, is caused by multiple triggers. The occurrence of cardiac arrhythmias in COVID-19 patients with myocardial involvement and a critical course is common. In this review, potential mechanisms, incidence, and treatment options for cardiac arrhythmias in COVID-19 patients will be provided by performing a literature research in MESH database and the National Library of Medicine. Common cardiac arrhythmias in COVID-19 patients were sinus tachycardia, atrial fibrillation (AF), ventricular tachycardia (VT), ventricular fibrillation (VF), atrioventricular block, sinusoidal block or QTc prolongation. AF was the most common heart rhythm disorder. About 10\% of COVID-19 patients develop new-onset AF and 23 to 33\% showed recurrence of $A F$ in patients with known AF. One retrospective trial revealed the incidence of VT or VF to be $5.9 \%$ in hospitalized patients. Both AF and VT are clearly associated with worse outcome. Several mechanisms such as hypoxia, myocarditis, myocardial ischemia, or abnormal host immune response, which induce cardiac arrhythmias, have been described. The effect of QT-prolonging drugs in inducing cardiac arrhythmias has become mitigated as these medications are no longer recommended. Acute management of cardiac arrhythmias in COVID-19 patients is affected by the reduction of exposure of health care personnel. More prospective data are desirable to better understand pathophysiology and consecutively adapt management.

Im Rahmen der sich aktuell weiterhin ausbreitenden Corona-Virus-Pandemie wurden bereits viele Millionen Menschen infiziert. Trotz zahlreicher oligosymptomatischer Fälle kann die Erkrankung einen schwerwiegenden oder tödlichen Verlauf nehmen und führt dann immer wieder zu einer Überlastung des Gesundheitssystems. COVID-19 kann durch unterschiedliche Pathomechanismen zu einer Affektion des Myokards führen, was wiederum das Entstehen von Rhythmusstörungen begünstigt. Dieser Artikel beschreibt Inzidenzen, potentielle Mechanismen sowie Behandlungsoptionen für Arrhythmien bei Patienten mit COVID-19-Erkrankung. Es erfolgte eine Literaturrechersche mittels MESH Database und PubMed. Die häufigsten Arrhythmien bei Patienten mit COVID-19 waren Sinustachykardie, received

May 10, 2021

accepted after revision

August 9, 2021 (c) 2021. Thieme. All rights reserved. Georg Thieme Verlag KG,

Rüdigerstraße 14,

70469 Stuttgart, Germany
DOI https://doi.org/

10.1055/a-1581-6881.

ISSN 0720-9355. 
Vorhofflimmern, ventrikuläre Tachykardien, Kammerflimmern, QTc-Verlängerung oder sinuatriale und atrioventrikuläre Blockierungen. Vorhofflimmern ist die häufigste Rhythmusstörung bei Patienten mit COVID-19. Ca. 10 Prozent der Patienten zeigen im Rahmen der Erkrankung neu aufgetretenes Vorhofflimmern und 23-33 Prozent ein Rezidiv von bereits vorbekanntem Vorhofflimmern. Die Häufigkeit von ventrikulären Tachykardien oder Kammerflimmern wird in einer retrospektiven Studie mit 5.9 Prozent angegeben. Beide Rhythmusstörungen sind mit einem ungünstigen Verlauf assoziiert. Verschiedene Mechanismen, die zum Auftreten von Rhythmusstörungen bei Patienten mit COVID-19 führen, wurden beschrieben: Diese sind unter anderem Hypoxie, Myokarditis, myokardiale Ischämie oder eine überschießende Immunantwort. Seit QTc-Zeit verlängernde Medikamente nicht mehr zur Behandlung von COVID-19 empfohlen werden, ist dieser Mechanismus für die Entstehung von Rhythmusstörungen deutlich weniger relevant. Die Minimierung der Exposition des Klinikpersonals stellt ein relevanter Gesichtspunkt in der Akutbehandlung von Arrhythmien bei COVID-19 Patienten dar. Zum genaueren Verständnis der Pathopyhsiologie und Optimierung von Behandlungsstrategien bei Patienten mit Rhythmusstörungen und COVID-19 wären prospektive Daten wünschenswert.

\section{Introduction}

Since December 2019 the world has been confronted by a rapidly evolving pandemic which was named coronavirus disease-2019 (COVID-19). Millions of cases have been reported worldwide, which has an enormous impact both on global health and economy. ${ }^{1}$ The disease is caused by severe acute respiratory syndrome coronavirus-2 (SARSCoV-2), which was first described in Wuhan, Hubei Province, in China. ${ }^{2}$ Despite a high number of asymptomatic cases, this pandemic has repeatedly led to extraordinary overburdening of health care systems all over the world. Common symptoms are cough, fever, fatigue, myalgia, diarrhea, and also loss of smell and taste. ${ }^{3}$ However, approximately $20 \%$ of patients develop acute respiratory distress syndrome (ARDS) in combination with other organ dysfunctions. Mechanical ventilation is reported to become necessary in approximately $13 \%{ }^{4}$ However, the affectation of the respiratory system seems to be only one brick in the pathogenesis of COVID-19. The enormous impact of other viral diseases like influenza or Coxsackie on the cardiovascular system has been described earlier. ${ }^{5}$ On the one hand, preexisting cardiovascular diseases (CVDs) as heart failure or ischemic cardiomyopathy become worse or decompensate during the metabolic demand in the acute viral phase and are, by this manner, an independent risk factor. On the other hand, several viruses are known to show direct toxic effects both on the myocardial and the endocardial cell, leading to increased incidences of myocarditis, ventricular arrhythmias, and rupture of atherosclerotic plaques. ${ }^{6}$ In line with better-known viruses, CVD was also identified to be a risk factor for severe courses in COVID-19 patients. ${ }^{7}$ The actual worldwide case-fatality rate of all COVID-19 patients is estimated to be approximately 2 to $3 \%$; however, in patients with preexisting CVD, it is reported to be approximately $13.2 \%{ }^{8,9}$ Moreover, COVID-19 infection leads to cardiac injury, as it has been described in more common viral diseases, which is defined as an increase in high sensitivity troponin above the 99th percentile. ${ }^{1,10}$ Myocardial involvement in patients, infected by COVID-19, is associated with critical or fatal courses of the disease. One study revealed mortality rates up to $51 \%$ in this patient population compared with $4.5 \%$ of patients without elevated troponin levels. ${ }^{11}$ Furthermore, the admission to intensive care unit is much more common, if myocardial involvement is present. ${ }^{4}$ Both the mechanisms and etiologies of acute cardiac injury are multicausal, including myocarditis, acute myocardial infarction, deep vein thrombosis and consecutive pulmonary embolism, aggravation of preexisting CVDs and oxygen demand-supply mismatch due to ARDS combined with shock. ${ }^{9}$ The occurrence of cardiac arrhythmias in patients with cardiac injury and severe course of COVID-19 is common. A meta-analysis revealed an incidence of $30.1 \%$ in critically ill patients versus $2.8 \%$ in mild courses. ${ }^{12}$ In particular, ventricular life-threatening arrhythmias like ventricular fibrillation (VF) or ventricular tachycardia (VT) appeared in $5.9 \%$ of patients admitted to intensive care unit. ${ }^{13}$ In this review, we provide the potential mechanisms, incidence and treatment options of cardiac arrhythmias in patients with COVID-19.

\section{Methods}

We performed a search for literature by using MESH database and the National Library of Medicine (Pubmed.gov) from December 1, 2019, to April 6, 2021. Keywords were "Cardiac arrhythmia" and "COVID-19." All articles, classified as clinical trials, meta-analysis, randomized control trial, "review," or "systematic review," were evaluated. Case reports, books or unpublished documents remained unconsidered. Submission of the complete text in English was a condition for inclusion. 


\section{Results}

Our literature research revealed 65 articles from 2020 until March 2021. Finally, seven articles, including background literature, were evaluated.

Arrhythmic manifestations in patients with COVID-19 were sinus tachycardia, atrial fibrillation (AF), atrial flutter, ectopic atrial tachycardia, atrioventricular nodal reentry tachycardia, VT, VF, atrioventricular or sinusoidal block, or QTc prolongation (-Table 1). ${ }^{1}$ A survey performed by the Heart Rhythm Society, including 1,197 patients, revealed tachyarrhythmia to be the most common rhythm disorder in COVID-19 patients (21\%) besides sinus tachycardia, whereas VT or VF accounts for $4.8 \%{ }^{14}$ The diversity of arrhythmias suggests various potential mechanisms for arrhythmogenesis. Both extracardiac and intracardiac processes can lead to cardiac arrhythmias in COVID-19 patients: Direct viral toxicity of both lung and myocardial tissue triggering myocarditis, myocardial dysfunction and ARDS can account for hypoxia of the myocardium which in turn result in cardiac arrhythmias. However, an abnormal host immune response, commonly seen in COVID-19 patients, myocardial stress caused by pulmonary hypertension, electrolyte or volume disbalances and myocardial ischemia might also be common triggers in COVID-19 patients. $^{15}$ The various etiologies of cardiac arrhythmias are shown in -Fig. 1 and are summarized in -Table 2.

QT-prolonging drugs: Hydroxychloroquine, chloroquine, or azithromycin with or without hydroxychloroquine were named in several reviews to be a relevant reason for cardiac arrhythmias in the earlier phase of the pandemic due to its QT-prolonging characteristics. ${ }^{1,16,17}$ Since these medications
Table 1 Cardiac arrhythmias in COVID-19 patients

\begin{tabular}{|l|}
\hline Sinus tachycardia \\
\hline Supraventricular tachycardia \\
\hline Atrial fibrillation and atrial flutter \\
\hline Ventricular premature complexes and nonsustained VT \\
\hline Conduction disturbances (AVB/BBB) \\
\hline Polymorphic ventricular tachycardia (torsade de pointes) \\
\hline Unstable ventricular tachycardia or ventricular fibrillation \\
\hline
\end{tabular}

Abbreviations: AVB, atrioventricular block; BBB, bundle branch block; VT, ventricular tachycardia.

Table 2 Mechanisms of cardiac arrhythmias in COVID-19 patients

\begin{tabular}{|l|}
\hline Myocarditis \\
\hline Hypoxia caused by acute respiratory distress syndrome \\
\hline Abnormal systemic immune response \\
\hline Myocardial strain \\
\hline Electrolyte and volume imbalance \\
\hline Myocardial ischemia \\
\hline Systemic inflammatory response syndrome \\
\hline
\end{tabular}

are no longer recommended for the treatment of COVID-19, this potential etiology of cardiac arrhythmias can be neglected. ${ }^{18-21}$ Meanwhile, dexamethasone and remdesivir were identified to favor the course of COVID-19 patients, but

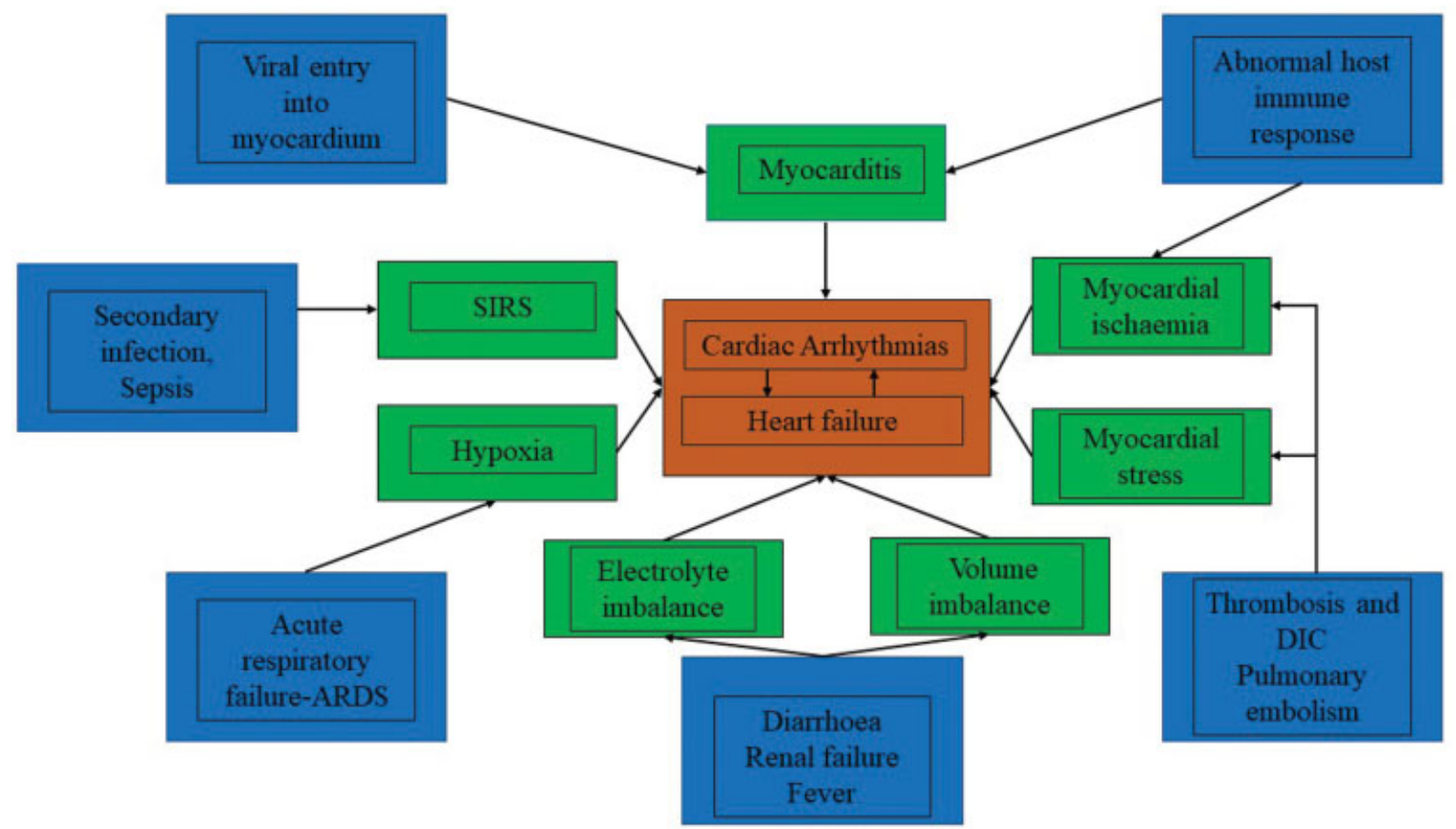

Fig. 1 Potential etiologies of cardiac arrhythmias in COVID-19 patients. ${ }^{1}$ ARDS, acute respiratory distress syndrome; DIC, disseminated intravascular coagulation; SIRS, systemic inflammatory response syndrome. 
they are not known to influence QT duration. ${ }^{22,23}$ Therefore, from our point of view, keeping in mind the potential QTprolonging arrhythmogenic potential of several medications is still of clinical relevance. However, this mechanism for cardiac arrhythmias does not any longer affect as many COVID-19 patients as it did in the first phase.

Hypoxia: Hypoxia as a result of acute respiratory failure in COVID-19 patients leads to hypoxia-mediated cardiomyocyte damage, similar to the pathophysiology in patients suffering from acute myocardial infarction. ${ }^{16}$ Due to a decrease in intracellular $\mathrm{pH}$, cytosolic calcium levels increase, which triggers early and late depolarization. Furthermore, hypoxia increases the concentration of extracellular potassium levels, leading to depressed thresholds for depolarization and is, by this manner, proarrhythmogenic. ${ }^{16}$

Abnormal host immune response: The course of COVID-19 has been described to pass three stages. Stage 1 is the early stage of infection, in which the typical symptoms of COVID-19 occur. Stage 2 is defined by the invasion and replication of the virus into pneumocytes, triggering an inflammatory immune response and in some cases ARDS. In some patients, the course converts into stage 3 , which is defined as a hyperinflammatory state, in which a cytokine storm triggers multiorgan dysfunction. ${ }^{24}$ Interleukin-6, tumor necrosis factor- $\alpha$ and interleukin-1 are some of the cytokines that lead to an immune cell-mediated injury of the myocardium. ${ }^{25}$ However, inflammatory cytokines also overactivate the cardiac sympathetic system, which aggravates preexisting coronary atherosclerosis, but is also able to induce prolonged QT duration and in turn might trigger lifethreatening arrhythmic events in patients with preexisting long-QT syndrome and COVID-19. ${ }^{16}$

Myocarditis: The occurrence of myocarditis in patients with COVID-19 has been described in previous studies. ${ }^{26}$ Several hypotheses, trying to explain the pathophysiology of myocarditis in COVID-19 patients, were recently published. First, direct viral penetration of the myocardial cell by utilizing the viral spike protein, which in turn binds to angiotensinconverting enzyme 2 receptor (ACE-2 receptor) on the myocardial cell membrane, leads to direct myocardial injury. ${ }^{16}$ Binding of the spike protein downregulates the ACE-2 receptor, which might trigger accumulation of angiotensin II and consecutively adverse remodeling of the myocardium. ${ }^{27}$ Both remodeling and viral penetration might disrupt electrical conduction and thus enhance arrhythmic risk. Another possible mechanism of inducing myocarditis and consecutively cardiac arrhythmias in COVID-19 patients is cellular-based. CD8+ T-lymphocytes as part of the systemic inflammation trigger inflammation of the myocytes. This mechanism is enhanced by a massive cytokine release, which increases the activity of T-lymphocytes, which in turn further releases cytokines. ${ }^{26} \mathrm{~A}$ vicious circle is created. Another trial revealed, that the amount of $\mathrm{CD} 14^{\mathrm{dim}} \mathrm{CD} 16^{+}$monocytes in peripheral blood of COVID-19 patients with severe coronary artery disease is remarkably decreased. ${ }^{28}$ These so-called nonclassical monocytes normally trigger cytokine release, which leads to systemic inflammation. The authors conclude that the decreased number of these nonclassical monocytes is due to migration into the heart and lung tissue, which in turn triggers tissue destruction and consecutively organ failure. Generally, the occurrence of cardiac arrhythmias in these acute inflammatory phases of COVID-19 myocarditis is finally due to gap junction dysfunction, electrophysiological remodeling with consecutive abnormal calcium handling and downregulation of potassium channels, leading to prolonged repolarization. ${ }^{29}$ However, cardiac arrhythmias can also be detected in the postacute phase of COVID-19, where residual myocardial scar might lead to reentry of arrhythmia. ${ }^{30}$

Myocardial ischemia: Acute myocardial infarction has also been reported to be triggered by COVID-19 infection. Activation of inflammatory cells within the preexisting atherosclerotic plaque with consecutive rupture might be the underlying mechanism. ${ }^{31}$ Cytokines such as interleukin-6 and tumor necrosis factor- $\alpha$, which are released during the hyperinflammatory stage, can cause a prothrombotic milieu and thus can lead to microvascular dysfunction. ${ }^{32}$ Another possible etiology of myocardial ischemia is an infectionmediated vasculitis, either by direct viral entry into the endothelial cells of the myocardial vessels or by an indirect immunological response. ${ }^{33}$

Right myocardial strain: Pressure load, leading to right myocardial strain, can be frequently seen in patients with COVID-19, either as a result of common pulmonary embolism in COVID-19 patients or as a consequence of ARDS, sepsis, and left-side heart failure. This pulmonary hypertension might trigger atrial tachyarrhythmias. ${ }^{34,35}$

Electrolyte derangements and volume imbalances: One case series study showed that electrolyte imbalances occur in $7.2 \%$ of COVID-19 patients. ${ }^{11}$ The incidence of acute kidney injury was reported to be approximately $27 \%{ }^{1}$ Fluid imbalances are also common in critically ill patients. The correlation between electrolyte or volume imbalances and triggering of preexisting proclivity to cardiac arrhythmias is well-established.

The management of cardiac arrhythmias in COVID-19 patients is as multifaceted as the underlying mechanisms and the arrhythmia itself. On the one hand, treatment in the era of COVID-19 should be as guideline-based as possible. On the other hand, due to the contagiousness of SARS-CoV-2, health care personnel should be protected and exposure should be reduced to a minimum. Under this aspect, the European Society of Cardiology (ESC) released a guidance paper for the diagnosis and management of CVD during the COVID-19 pandemic (available at: https://www.escardio. org/Education/COVID-19-and-Cardiology/ESCCOVID-19-

Guidance). In the following section, the management of the miscellaneous cardiac arrhythmias will be discussed.

$A F$ and atrial flutter: As stated earlier, $\mathrm{AF}$ is the most common heart rhythm disorder in COVID-19 patients ${ }^{14}: 23$ to $33 \%$ of COVID-19 patients with sepsis or ARDS caused by COVID-19 showed AF recurrence in patients with a history of AF and approximately $10 \%$ developed new-onset $\mathrm{AF}$, which is clearly associated with worse outcome. ${ }^{36}$ Several mechanisms have been described to possibly promote the occurrence of AF: hypoxia, which is frequently seen in ARDS patients; myocarditis; systemic inflammation; electrolyte disturbances, the use of inotropic agents, and sympathetic 
nervous system overactivity, which trigger AF or atrial flutter in patients who already show proclivity for these heart rhythm disorders. ${ }^{37}$ As in non-COVID patients, the decision tree for appropriate treatment of AF or atrial flutter dichotomizes into hemodynamically stable and unstable. If patients are unstable, immediate synchronized current cardioversion becomes necessary. ${ }^{17}$ In case of hemodynamic stability, the use of $\beta$-blocker or calcium channel blocker seems to be the first-line therapy, unless there are contraindications. Heart rate control can be further reached by the addition of digoxin/digitoxin. ${ }^{17}$ Amiodarone can also be used either for heart rate control or for restoration of sinus rhythm. Its QT-prolonging side effect should be carefully monitored in COVID-19 patients. However, the impact of this special side effect has been mitigated by the withdrawal of other potential QT-prolonging drugs for the treatment of COVID-19. Furthermore, common underlying triggers such as hypoxia in ARDS patients, hypokalemia and hypomagnesemia, metabolic acidosis, catecholamine infusion, hypervolemia, hypovolemia, and bacterial superinfection should be addressed. ${ }^{17}$ The need for transthoracic or transesophageal cardiac ultrasound should always be balanced against the possible contamination of health care personnel and equipment. Anticoagulation after onset of AF for the prevention of cerebral or systemic embolism should be encouraged in any case of sepsis or heart failure. After recovery from COVID-19, heart rhythm should be reassessed and appropriate treatment including rhythm control and anticoagulation should be reevaluated in accordance with the $\mathrm{CHA}_{2} \mathrm{DS}_{2}$-VASc score and current guidelines. ${ }^{38}$

Interestingly, apart from the acute treatment, another impact of the COVID-19 pandemic and consecutive national lockdown has been described in a Danish trial. ${ }^{39}$ There was a $47 \%$ drop of incidence of newly diagnosed AF during lockdown in March and April 2020 compared with 2019. The authors conclude that consecutively a high number of newonset AF cases remained undetected leading to poorer outcomes and increased complications in this patient collective. Therefore, an ECG diagnostic should be promptly performed in every hospitalized patient ( - Table 3 ).

Ventricular tachycardia or VF: As stated earlier, one single-center retrospective trial from Wuhan revealed the incidence of sustained VT or VF to be $5.9 \%$ in hospitalized COVID-19 patients. Cardiac injury was found in $27.8 \%$ of the patients, defined by elevated troponin levels, which was significantly associated with an increased rate of malignant ventricular arrhythmias (17.3 vs. $1.5 \%, p<0.001) .{ }^{40}$ Another retrospective cohort study of 1,284 patients measured cardiac troponin levels in 1,159 patients on admission. The incidence of cardiac injury was $14.7 \%$ (170 patients). Six of these patients showed VT or VF and all of them died. ${ }^{41}$ Acute and severely diseased COVID-19 patients commonly develop cofactors such as electrolyte imbalances (hypokalemia and hypomagnesemia), fever, hypoxia or an inflammatory state, which might trigger VT or VF, both de novo in patients with preexisting heart disease and a history of malignant ventricular arrhythmias. ${ }^{17}$ Since the application of QT-prolonging immunomodulatory drugs, such as
Table 3 Clinical care of COVID-19 patients in regard to cardiac arrhythmias

\begin{tabular}{l}
12 lead ECG and rhythm strip in all hospitalized \\
COVID-19 patients \\
ECG monitoring of COVID-19 patients in case of: \\
- Nonsustained VTs \\
- New-onset atrial fibrillation and flutter \\
- New AVB/BBB \\
- New QTc prolongation $>0.5 \mathrm{~s}$ \\
\hline ICU, availability of circulation support \\
- Sustained VT, unstable VT \\
- Bradycardia due to AV block \\
- Torsade de pointes tachycardia or ventricular \\
fibrillation
\end{tabular}

Abbreviations: AV, atrioventricular; AVB, atrioventricular block; BBB, bundle branch block; ECG, electrocardiography; ICU, intensive care unit; VT, ventricular tachycardia.

hydroxychloroquine, azithromycin and lopinavir/ritonavir, are no longer recommended for the treatment of COVID-19 patients, the development of acquired QT prolongation and consecutively torsade de pointes tachycardias has lost its crucial clinical relevance. However, one trial reported QTprolongation and VT in COVID-19 patients in the absence of appropriate drugs. ${ }^{42}$ The management of COVID-19 patients suffering from VT or VF is, in most instances, similar to allcomers with malignant arrhythmias. However, deferrable examinations and consecutive exposition of health care employees should be avoided. In case of VF or pulseless VT defibrillation should be performed. If hemodynamically unstable patients with VT present themselves, synchronized electrical cardioversion is the preferred treatment. ${ }^{43}$ Intravenous amiodarone and $\beta$-blocker are the treatment of choice in cases of recurrent VT or electrical storm. Sedation and intubation become necessary in refractory cases. Ablation can be considered even in the acute phase as bailout therapy. ${ }^{44}$ However, potential benefit should be balanced against potential viral contamination. Underlying reversible triggers such as hypoxia, hypovolemia, electrolyte imbalances, metabolic acidosis and catecholamine infusions should be corrected. An underlying ischemic heart disease or an acute coronary syndrome should be treated properly. ${ }^{43}$ QT-prolongation and torsade de pointes should be treated by withdrawal of all QT-prolonging drugs, intravenous magnesium supplementation, correction of potassium levels and avoidance of bradycardia by withdrawal of bradycardic agents and, if necessary, by temporary pacing. Treatment of life-threatening arrhythmias should be performed in a heart center with availability of circulation support devices (- Table 3). ${ }^{43}$ After recovery from COVID19 , both indication of an implantable cardioverter-defibrillator (ICD) and ablation of VT should be reevaluated and detailed examination should be performed to detect or exclude structural heart disease, as the acute infection may be a reversible trigger. ${ }^{43}$ Wearable ICD vests can be considered in cases of unclear reversibility of cardiac involvement. ${ }^{1}$ 
Bradyarrhythmias: The occurrence of an atrioventricular block can be seen in up to $12 \%$ of cardiac arrhythmias in COVID-19 patients and is associated with worse outcome. ${ }^{45}$ A retrospective study of 756 COVID-19 patients revealed in a multivariate analysis the risk of death to be higher in the presence of atrioventricular block (odds ratio $=2.61$, $p=0.002) .{ }^{46}$ The mechanism behind sinus or atrioventricular block is not clear. However, severe hypoxia, inflammatory injury of the sinus node and the conduction system or an involvement of the conduction or impulse-forming system in patients with myocarditis is most likely. ${ }^{17}$ Isoprenaline and atropine can be administered before temporary pacemaker implantation. After recovery from COVID-19, the indication for permanent pacemaker needs to be reevaluated. ${ }^{1}$

\section{Conclusion}

Cardiac arrhythmias, especially AF and life-threatening VT, may be the consequence of various impacts of COVID-19 infection. Several mechanisms, that induce cardiac arrhythmias have been described. Since QT-prolonging antiviral medication has no longer been recommended, the role of drug-induced torsade de pointes has become less relevant. Triggers of cardiac arrhythmias in COVID-19 patients, such as hypoxia, electrolyte imbalance and fever are common and have to be prevented. The severity of arrhythmias should trigger the intensity of clinical care. To minimize exposure of health care personnel is of crucial relevance, which in turn affects acute management.

The occurrence of cardiac arrhythmias is strongly associated with myocardial injury, is more common in a critical care setting and leads to worse outcome. For this reason, more prospective data are desirable to better understand the pathophysiology and improve management.

\section{Conflict of Interest}

The authors declare that they have no conflict of interest.

\section{References}

1 Dherange P, Lang J, Qian P, et al. Arrhythmias and COVID-19: a review. JACC Clin Electrophysiol 2020;6(09):1193-1204

2 Jee Y. WHO International Health Regulations Emergency Committee for the COVID-19 outbreak. Epidemiol Health 2020;42: e2020013

3 Stokes EK, Zambrano LD, Anderson KN, et al. Coronavirus disease 2019 case surveillance - United States, January 22-May 30, 2020. MMWR Morb Mortal Wkly Rep 2020;69(24):759-765

4 Wang D, Hu B, Hu C, et al. Clinical characteristics of 138 hospitalized patients with 2019 novel coronavirus-infected pneumonia in Wuhan, China. JAMA 2020;323(11):1061-1069

5 Vardeny O, Madjid M, Solomon SD. Applying the lessons of influenza to COVID-19 during a time of uncertainty. Circulation 2020;141(21):1667-1669

6 Kwong JC, Schwartz KL, Campitelli MA, et al. Acute myocardial infarction after laboratory-confirmed influenza infection. $\mathrm{N}$ Engl J Med 2018;378(04):345-353

7 Petrilli CM, Jones SA, Yang J, et al. Factors associated with hospital admission and critical illness among 5279 people with coronavirus disease 2019 in New York City: prospective cohort study. BMJ 2020;369:m1966
8 Dong E, Du H, Gardner L. An interactive web-based dashboard to track COVID-19 in real time. Lancet Infect Dis 2020;20(05): 533-534

9 Dou Q, Wei X, Zhou K, Yang S, Jia P. Cardiovascular manifestations and mechanisms in patients with COVID-19. Trends Endocrinol Metab 2020;31(12):893-904

10 Rath D, Petersen-Uribe Á, Avdiu A, et al. Impaired cardiac function is associated with mortality in patients with acute COVID-19 infection. Clin Res Cardiol 2020;109(12):1491-1499

11 Shi S, Qin M, Shen B, et al. Association of cardiac injury with mortality in hospitalized patients with COVID-19 in Wuhan, China. JAMA Cardiol 2020;5(07):802-810

12 Wen W, Zhang H, Zhou M, et al. Arrhythmia in patients with severe coronavirus disease (COVID-19): a meta-analysis. Eur Rev Med Pharmacol Sci 2020;24(21):11395-11401

13 Driggin E, Madhavan MV, Bikdeli B, et al. Cardiovascular considerations for patients, health care workers, and health systems during the COVID-19 pandemic. J Am Coll Cardiol 2020;75(18): 2352-2371

14 Gopinathannair R, Merchant FM, Lakkireddy DR, et al. COVID-19 and cardiac arrhythmias: a global perspective on arrhythmia characteristics and management strategies. J Interv Card Electrophysiol 2020;59(02):329-336

15 Bhatla A, Mayer MM, Adusumalli S, et al. COVID-19 and cardiac arrhythmias. Heart Rhythm 2020;17(09):1439-1444

16 Lazzerini PE, Boutjdir M, Capecchi PL. COVID-19, arrhythmic risk, and inflammation: mind the gap!. Circulation 2020;142 (01):7-9

17 Manolis AS, Manolis AA, Manolis TA, Apostolopoulos EJ, Papatheou D, Melita H. COVID-19 infection and cardiac arrhythmias. Trends Cardiovasc Med 2020;30(08):451-460

18 Self WH, Semler MW, Leither LM, et al; National Heart, Lung, and Blood Institute PETAL Clinical Trials Network. Effect of hydroxychloroquine on clinical status at 14 days in hospitalized patients with COVID-19: a randomized clinical trial. JAMA 2020;324(21): 2165-2176

19 Cavalcanti AB, Zampieri FG, Rosa RG, et al; Coalition COVID-19 Brazil I Investigators. Hydroxychloroquine with or without azithromycin in mild-to-moderate COVID-19. N Engl J Med 2020;383 (21):2041-2052

20 Tang W, Cao Z, Han M, et al. Hydroxychloroquine in patients with mainly mild to moderate coronavirus disease 2019: open label, randomised controlled trial. BMJ 2020;369:m1849

21 Horby P, Mafham M, Linsell L, et al; RECOVERY Collaborative Group. Effect of hydroxychloroquine in hospitalized patients with COVID-19. N Engl J Med 2020;383(21):2030-2040

22 Beigel JH, Tomashek KM, Dodd LE, et al; ACTT-1 Study Group Members. Remdesivir for the treatment of COVID-19 - final report. N Engl J Med 2020;383(19):1813-1826

23 Sterne JAC, Murthy S, Diaz JV, et al; WHO Rapid Evidence Appraisal for COVID-19 Therapies (REACT) Working Group. Association between administration of systemic corticosteroids and mortality among critically ill patients with COVID-19: a metaanalysis. JAMA 2020;324(13):1330-1341

24 Atri D, Siddiqi HK, Lang JP, Nauffal V, Morrow DA, Bohula EA. COVID-19 for the cardiologist: basic virology, epidemiology, cardiac manifestations, and potential therapeutic strategies. JACC Basic Transl Sci 2020;5(05):518-536

25 Puntmann VO, Taylor PC, Barr A, Schnackenburg B, Jahnke C, Paetsch I. Towards understanding the phenotypes of myocardial involvement in the presence of self-limiting and sustained systemic inflammation: a magnetic resonance imaging study. Rheumatology (Oxford) 2010;49(03):528-535

26 Siripanthong B, Nazarian S, Muser D, et al. Recognizing COVID-19related myocarditis: the possible pathophysiology and proposed guideline for diagnosis and management. Heart Rhythm 2020;17 (09):1463-1471 
27 Vaduganathan M, Vardeny O, Michel T, McMurray JJV, Pfeffer MA Solomon SD. Renin-angiotensin-aldosterone system inhibitors in patients with COVID-19. N Engl J Med 2020;382(17):1653-1659

28 Mueller KAL, Langnau C, Günter M, et al. Numbers and phenotype of non-classical CD14 ${ }^{\mathrm{dim}} \mathrm{CD} 16^{+}$monocytes are predictors of adverse clinical outcome in patients with coronary artery disease and severe SARS-CoV-2 infection. Cardiovasc Res 2021;117(01):224-239

29 Tse G, Yeo JM, Chan YW, Lai ET, Yan BP. What is the arrhythmic substrate in viral myocarditis? Insights from clinical and animal studies. Front Physiol 2016;7:308

30 Peretto G, Sala S, Rizzo S, et al. Arrhythmias in myocarditis: state of the art. Heart Rhythm 2019;16(05):793-801

31 Musher DM, Abers MS, Corrales-Medina VF. Acute infection and myocardial infarction. N Engl J Med 2019;380(02):171-176

32 Hunt BJ. Bleeding and coagulopathies in critical care. N Engl J Med 2014;370(09):847-859

33 Varga Z, Flammer AJ, Steiger P, et al. Endothelial cell infection and endotheliitis in COVID-19. Lancet 2020;395(10234):1417-1418

34 Klok FA, Kruip MJHA, van der Meer NJM, et al. Incidence of thrombotic complications in critically ill ICU patients with COVID-19. Thromb Res 2020;191:145-147

35 Wanamaker B, Cascino T, McLaughlin V, Oral H, Latchamsetty R, Siontis KC. Atrial arrhythmias in pulmonary hypertension: pathogenesis, prognosis and management. Arrhythm Electrophysiol Rev 2018;7(01):43-48

36 Boriani G, Fauchier L, Aguinaga L, et al; ESC Scientific Document Group. European Heart Rhythm Association (EHRA) consensus document on management of arrhythmias and cardiac electronic devices in the critically ill and post-surgery patient, endorsed by Heart Rhythm Society (HRS), Asia Pacific Heart Rhythm Society (APHRS), Cardiac Arrhythmia Society of Southern Africa (CASSA), and Latin American Heart Rhythm Society (LAHRS). Europace 2019;21(01):7-8

37 Russo V, Rago A, Carbone A, et al. Atrial fibrillation in COVID-19: from epidemiological association to pharmacological implications. J Cardiovasc Pharmacol 2020;76(02):138-145
38 Hindricks G, Potpara T, Dagres N, et al; ESC Scientific Document Group. 2020 ESC Guidelines for the diagnosis and management of atrial fibrillation developed in collaboration with the European Association for Cardio-Thoracic Surgery (EACTS): The Task Force for the diagnosis and management of atrial fibrillation of the European Society of Cardiology (ESC) Developed with the special contribution of the European Heart Rhythm Association (EHRA) of the ESC. Eur Heart J 2021;42(05):373-498

39 Holt A, Gislason GH, Schou M, et al. New-onset atrial fibrillation: incidence, characteristics, and related events following a national COVID-19 lockdown of 5.6 million people. Eur Heart J 2020;41 (32):3072-3079

40 Guo T, Fan Y, Chen M, et al. Cardiovascular implications of fatal outcomes of patients with coronavirus disease 2019 (COVID-19). JAMA Cardiol 2020;5(07):811-818

41 Si D, Du B, Ni L, et al. Death, discharge and arrhythmias among patients with COVID-19 and cardiac injury. CMAJ 2020;192(28): E791-E798

42 Toubiana J, Poirault C, Corsia A, et al. Kawasaki-like multisystem inflammatory syndrome in children during the COVID-19 pandemic in Paris, France: prospective observational study. BMJ 2020;369:m2094

43 Desai AD, Boursiquot BC, Melki L, Wan EY. Management of arrhythmias associated with COVID-19. Curr Cardiol Rep 2020; 23(01):2

44 Heinzmann D, Schibilsky D, Gramlich M, et al. Ablation of an electrical storm in a patient with giant cell myocarditis using continuous flow left ventricular assist device and percutaneous right ventricular assist device. Int J Cardiol 2016; 209:84-86

45 Wang Y, Wang Z, Tse G, et al. Cardiac arrhythmias in patients with COVID-19. J Arrhythm 2020;36(05):827-836

46 McCullough SA, Goyal P, Krishnan U, Choi JJ, Safford MM, Okin PM. Electrocardiographic findings in coronavirus disease-19: insights on mortality and underlying myocardial processes. J Card Fail 2020;26(07):626-632 